\title{
Diffraction Removal in an Image-based BRDF Measurement Setup
}

\author{
Antoine Lucat; Univ. Bordeaux, CNRS (LP2N), Institut d'Optique Graduate School, INRIA Bordeaux Sud-Ouest; Talence, France \\ Ramon Hegedus; Department of Cognitive Science; Tubingen, Germany \\ Romain Pacanowski; CNRS (LP2N), Institut d'Optique Graduate School, Univ. Bordeaux, INRIA Bordeaux Sud-Ouest; Talence, \\ France
}

\begin{abstract}
Material appearance is traditionally represented through its Bidirectional Reflectance Distribution Function (BRDF), quantifying how incident light is scattered from a surface over the hemisphere. To speed up the measurement process of the BRDF for a given material, which can necessitate millions of measurement directions, image-based setups are often used for their ability to parallelize the acquisition process: each pixel of the camera gives one unique configuration of measurement. With highly specular materials, the High Dynamic Range (HDR) imaging techniques are used to acquire the whole BRDF dynamic range, which can reach more than 10 orders of magnitude. Unfortunately, HDR can introduce star-burst patterns around highlights arising from the diffraction by the camera aperture. Therefore, while trying to keep track on uncertainties throughout the measurement process, one has to be careful to include this underlying diffraction convolution kernel. A purposely developed algorithm is used to remove most part of the pixels polluted by diffraction, which increase the measurement quality of specular materials, at the cost of discarding an important amount of BRDF configurations (up to $90 \%$ with specular materials). Finally, our setup succeed to reach a $1.5^{\circ}$ median accuracy (considering all the possible geometrical configurations), with a repeatability from $1.6 \%$ for the most diffuse materials to $5.5 \%$ for the most specular ones. Our new database, with their quantified uncertainties, will be helpful for comparing the quality and accuracy of the different experimental setups and for designing new image-based BRDF measurement devices.
\end{abstract}

\section{Motivation and Related Work}

Since the seminal work of Marschner [1] or Matusik et al. [2] on BRDF acquisition, a lot of devices and measurement setups have been developed to acquire the appearance of materials.

For the last two decades, most researchers from the Vision or CG communities have focused on acquiring more complex appearance functions, such as spatially-varying BRDF (e.g., [3, 4]) to Bidirectional Texture function or tried to reduce setup complexity or cost (e.g., [5]).

Unfortunately, most setups or available measurements data are hard to compare because they rely on qualitative validation instead of a quantitative one. For example, many papers (e.g., [6]) that try to fit BRDF analytical models discard measurements at arbitrary grazing angles because they seem unreliable.

We believe that new available data with their quantified uncertainties will be helpful for the research community, especially in order to compare the quality and accuracy of the different experimental setups and even to help other research teams to de-

\begin{tabular}{|l|l|}
\hline$\omega_{o} \rightarrow\left(\theta_{o}, \phi_{o}\right)$ & $\begin{array}{l}\text { Camera view vector in camera } \\
\text { space and angles in the surface } \\
\text { tangent frame }\end{array}$ \\
\hline$\omega_{i} \rightarrow\left(\theta_{i}, \phi_{i}\right)$ & $\begin{array}{l}\text { Incident light vector in camera } \\
\text { space and angles in the surface } \\
\text { tangent frame }\end{array}$ \\
\hline $\begin{array}{l}\rho\left(\theta_{i}, \theta_{o}, \Delta \phi\right) \\
\text { where } \Delta \phi=\left|\phi_{o}-\phi_{i}\right|\end{array}$ & Isotropic BRDF function \\
\hline$\rho_{r}, \rho_{g}, \rho_{b}$ & BRDF RGB values \\
\hline$S$ & $\begin{array}{l}\text { Sphere on which the BRDF is } \\
\text { measured }\end{array}$ \\
\hline$p_{S}$ & Point on $S$ \\
\hline $\mathbf{n}_{S}$ & Normal vector of $p_{S}$ \\
\hline$\left(R_{S}, C_{S}\right)$ & Radius and position of the sphere \\
\hline$f$ & Focal length \\
\hline$\phi_{\text {stage }}$ & Angle of the rotation stage \\
\hline
\end{tabular}

Table 1: Mathematical notations used in this paper.

velop their devices. Furthermore, uncertainty values can be useful to guide approximation techniques (e.g., Levenberg-Marquardt, SQP, Weighted Least-Square), which are necessary to obtain parameters of analytical models.

In order to compute precisely the resulting measurement uncertainties, an important effect that is always neglected in HDR image-based BRDF measurement setups is the influence of diffraction in the imaging process. With high frequency materials, HDR imaging techniques used to acquire the whole BRDF dynamic range can reach up to 10 orders of magnitude. Unfortunately, HDR can introduce star-burst patterns around highlights (cf. Fig. 3) arising from the diffraction on the camera aperture. The spatial extent of this effect can be very wide and alters pixels values, making them unreliable for measurement purposes. If not properly taken into account, this measurement artifact can widen artificially the specular lobe, which then do not represent the real BRDF any more. Lucat and al. [7] developed a method to remove the pixels most affected by diffraction, limiting the effect, at the cost of discarding a lot of BRDF configurations. In this paper, we illustrate the impact of the diffraction removal algorithm on our BRDF measurement setup.

Finally, this paper presents a new set of isotropic BRDF measurements with their radiometric and geometric uncertainties acquired from our imaging device. The uncertainties are derived from a model that takes into account the different sources of errors (e.g., focal length, mechanical, ...). Therefore, we illus- 


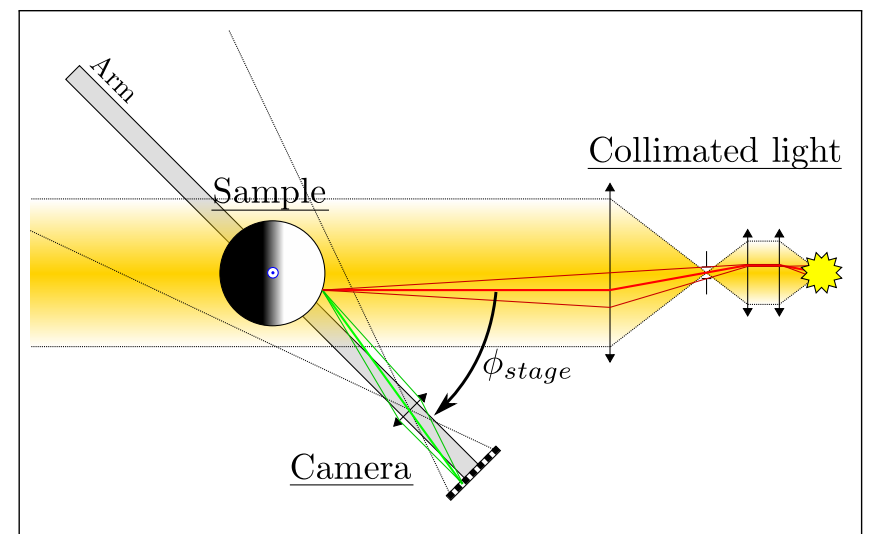

Figure 1: Our BRDF measurement setup: a rotation arm rotates a camera around the spherical sample. Each position of the arm gives a different angle between the view direction and the light direction, and each pixel of the camera intersect the surface with a unique tangent surface configuration.

\begin{tabular}{|c|l|}
\hline $\begin{array}{c}\text { Name } \\
\text { Material }\end{array}$ & $\begin{array}{l}\text { Constructor parameter(s) } \\
\text { Calibrated parameter }(\mathrm{s})\end{array}$ \\
\hline $\begin{array}{c}\text { Light source } \\
\text { Xion Xenon R } 180\end{array}$ & $\begin{array}{l}\text { Spectrum } \\
\text { Collimation: } 2.5 \mathrm{mrad}\end{array}$ \\
\hline $\begin{array}{c}\text { Sphere } \\
\text { Material dependant }\end{array}$ & $\begin{array}{l}\text { Radius: around } 3 \mathrm{~cm} \\
\text { Center-to-camera: } 67 \mathrm{~cm}\end{array}$ \\
\hline $\begin{array}{c}\text { Rotation stage } \\
\text { Zaber T-RS60 }\end{array}$ & Repeatability: $0.4 \mathrm{mrad}$ \\
\hline $\begin{array}{c}\text { Camera sensor } \\
\text { Jenoptik ProgRes C14 Plus }\end{array}$ & $\begin{array}{l}\text { Resolution: } 1024 \mathrm{x} 1360 \\
\text { Pixel: } 6.45 \mu \mathrm{m} \times 6.45 \mu \mathrm{m}\end{array}$ \\
\hline $\begin{array}{l}\text { Camera Lens (pinhole equiva- } \\
\text { lence) }\end{array}$ & F-number: 11 \\
Linos MeVis-C 50mm & Focal length: $53 \mathrm{~mm}$ \\
\hline
\end{tabular}

Table 2: Setup hardware configuration

trate how the measurement quality behaves with different types of materials. Finally, we discuss the limitations of our current measurement method and offer possible improvements for it.

\section{BRDF Setup}

Our BDRF measurement setup (cf. Fig. 1) is based on the one introduced by Matusik et al. [2]. It is composed of a fixed collimated light source that illuminates uniformly a sphere covered by the material to acquire. The sphere sits at the center of a rotation stage that rotates an arm at the end of which a scientificgrade camera is firmly mounted. The list of the currently used material and its main characteristics is provided in Table 2. Since the sphere and the camera are fixed on the rotation stage, they are always facing each other regardless of the rotation step.

\section{Measurement Method}

This setup aims to capture isotropic BRDFs. As defined by Nicodemus [8], the BRDF $\rho$ is a positive quantity expressed in $s r^{-1}$ (inverse steradian) representing how a material reflects angularly the light. For a given point $p_{S}$ on a surface $S, \rho$ is defined as the ratio of the differential reflected radiance $L_{r}$ and the differential irradiance $d E$ :

$$
\rho\left(\theta_{i}, \theta_{o}, \Delta \phi\right)=\frac{L_{r}\left(\theta_{o}, \phi_{o}\right)}{d E\left(\theta_{i}, \phi_{i}\right)}
$$

where all angles $\left(\theta_{i}, \theta_{o}, \Delta \phi\right)$ are expressed in the local tangent frame of the surface orientated with a normal vector $\mathbf{n}_{S}$. Our setup therefore needs the same number of degrees of freedom. The first one is given by the rotation stage, providing an angle $\phi_{\text {stage }}$ between the light and the view directions. The last two degrees of freedom arise from the fact that each camera pixel corresponds to a unique surface normal $\mathbf{n}_{S}$, that finally describes the whole hemisphere due to the use of a spherical object. In fact, one can show that for a given rotation step, all the angular configurations correspond to a unique slice of the Rusinkiewicz BRDF parametrization [9].

When using a broadband white light source with an RGBsensor, the measured BRDF can be seen as an angular function returning an RGB value:

$$
\rho\left(\theta_{i}, \theta_{o}, \Delta \phi\right) \rightarrow\left(\rho_{r}, \rho_{g}, \rho_{b}\right)
$$

Unfortunately, using a single time exposure is often not sufficient to capture the whole dynamic range of a BRDF, that can range up to 10 orders of magnitude. In order to solve for this issue, we use a technique known as HDR imaging, where multiple pictures of different exposures are merged to form a synthetic image of increased dynamic range. Then, for each rotation step, each pixel corresponds to a unique angular configuration of the BRDF on the surface of the sample, and the corresponding HDR color values relative to the incident light gives the BRDF color values via equation (1).

\section{Calibration}

In order to track precisely each ray in the scene and its radiometric properties, a precise calibration is needed. Conceptually, the aim is to be able to simulate accurately our setup in order to understand the path of each ray, while tracking each uncertainty (discussed in the Uncertainies Model section). The camera space has been chosen to represent the whole scene since every calibration is done using the camera. The calibration procedure (cf. Fig. 2) consists of five major steps: (i) camera calibration, (ii) mirror sphere calibration, (iii) light directions calibration, (iv) light uniformity calibration, (v) spherical sample calibration.

(i) Camera calibration. The goal of this calibration is to compute the view ray $\omega_{o}$ for each pixel. For that purpose, we used the calibration procedure for a pinhole model, from the Caltech library [10]. Although the library allows for a complex description of the camera, simply considering a perfect pinhole camera turned to be sufficient to obtain a sub-pixel accuracy. Therefore, the calibration returns a unique value: the focal length $f$ of the pinhole model. Radiometrically, we need to ensure that the camera response is linear with respect to the incoming ray intensity. A precise characterization showed that, for our 14-bit coded pixel values, the range from 300 to 15800 is linear below the noise level. This range of values has also the benefit that the only significant source of noise is the photon shot noise. Finally, due to longer exposure times some hot pixels can appear in the images, which need to be discarded through outlier pixel detection.

(ii \& v) Sphere calibration. Given the radius of a sphere $R_{S}$ and its uncertainty (from the manufacturer), this calibration step aims to find the $3 \mathrm{D}$ position $C_{S}$ of the sphere center in the camera space. Our camera being well described by a pinhole model, imaging a sphere is done through a perspective projection. Mathematically, it turns out that, onto the camera sensor, the 


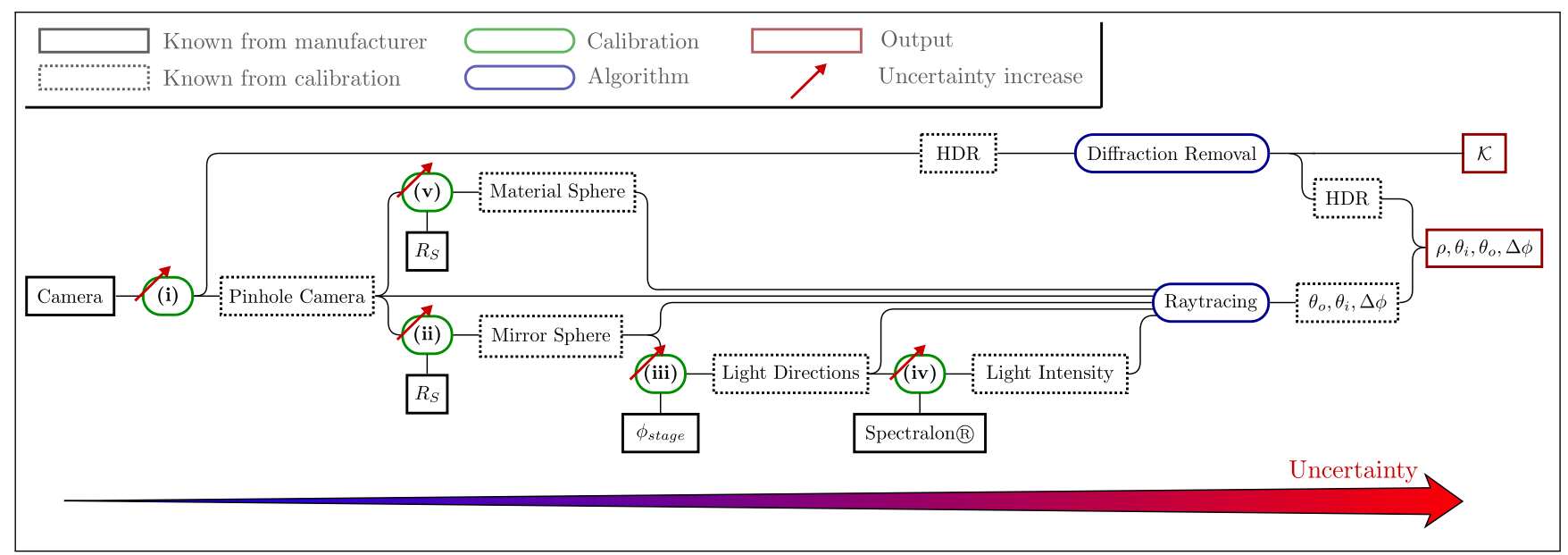

Figure 2: Relationship between the different parameters of our setup. Each physical element undergoes a calibration process (cf. (i-v) in the calibration section), which is then used to compute all BRDF configurations. Throughout this process, each uncertainty adds up to the final measured BRDF value according to equation (3).

sphere contour describes a perfect ellipse. Fitting this ellipse allows us to recover the sphere center $C_{S}$, with a very good accuracy $(\sim 50 \mu \mathrm{m})$.

(iii) Light directions calibration. For each rotation step of the arm, we need to know the light direction in the camera space. First, we place a calibrated mirror sphere (calibrated via (ii)) in front of the camera. The light-spot position on the sphere gives the light direction $\omega_{i}$ from the law of reflection. This procedure is repeated every $5^{\circ}$ across the whole arm revolution. Since light directions do not offset much from a perfect linear rotation, the intermediate angular positions can be deduced by interpolation.

(iv) Light uniformity calibration. The collimated light does not lit the sphere with a uniform intensity across the beam. In order to correct it, we set a $8^{\circ}$ offset between the light and the camera (the smallest before occlusion of the beam by the camera),

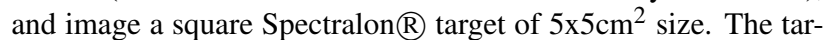
get contour on the image allows us to recover the $3 \mathrm{D}$ configuration of the target through an optimization process, with a sub-pixel accuracy. The position of the target allows then to create a direct mapping between the image values and the intensity repartition within the light beam. Using this material to map the beam intensity is ideal since its reflectance is almost Lambertian at normal angles.

This entire procedure leads to a fully characterized setup, both geometrically and radiometrically. For each pixel, it is then possible to compute the intersection with the sphere, the light direction and intensity at this point, and the outgoing view direction.

\section{Diffraction Treatment}

HDR imaging is inevitable when it comes to capturing high dynamic range BRDFs. In order to minimize optical aberrations, we set the camera f-number to 11 , a number that appears to be ideal regarding the trade-off between the amount of light captured, the optical aberrations, the depth of field, and the diffraction effect. Yet, when the picture has strong reflections, typical star-burst patterns can appear due to diffraction through the lens diaphragm (cf. Fig. 3). This is especially prominent with specular materials where the dynamic range goes beyond 10 orders of magnitude.

The effect of diffraction on the image is described by a con-

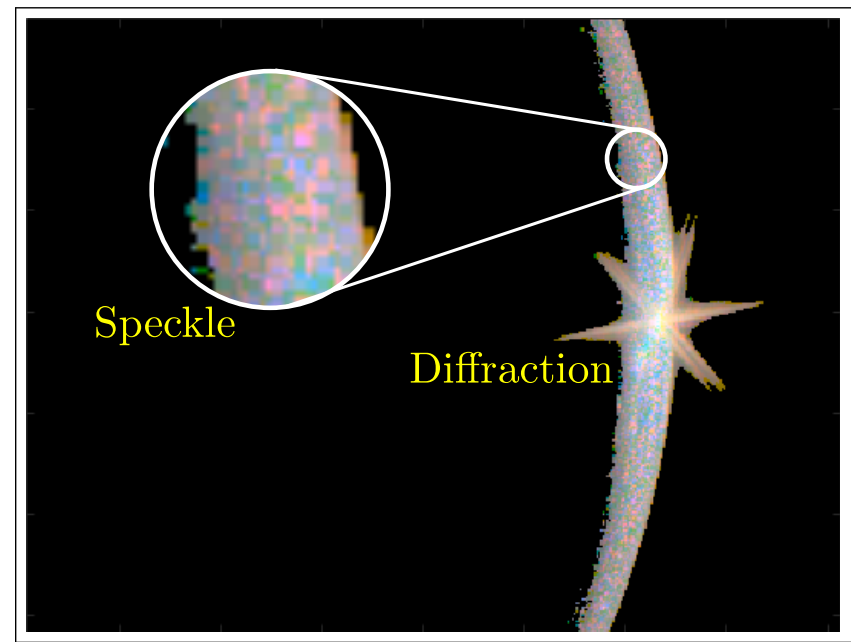

Figure 3: Portion of a tone-mapped HDR image captured during the process (at $\phi_{\text {stage }}=168^{\circ}$ ). In this picture, the dynamic range is 6 decades, enhancing the effect of diffraction: the values of the pixels were diffraction occurs are not reliable, then have to be taken into account. This picture shows also a typical colored noise, due to the white light speckle [11].

volution between the original unblurred image and a kernel called Point Spread Function (PSF). Because of this convolution, pixel values become no longer reliable and trying to recover the original signal via deconvolution algorithm grants no boundary on the recovered picture uncertainty. Moreover, it is not conceivable to accept this diffraction effect since it can highly perturb the measured BRDF values. Lucat and al. [7] proposed a method to detect pixels that are polluted by diffraction beyond a certain userdefined threshold. We use the method proposed in their article since it is able to give a very accurate analytical PSF kernel from a curved polygon model of the diaphragm, which accurately fits the diaphragm shape of our Linos camera lens.

Given the picture with diffraction and the PSF, the algorithm outputs a residual kernel (noted $\mathscr{K}$ ) and a mask (cf. Fig. 4) identifying which pixel can be considered affected by diffraction. Ac- 


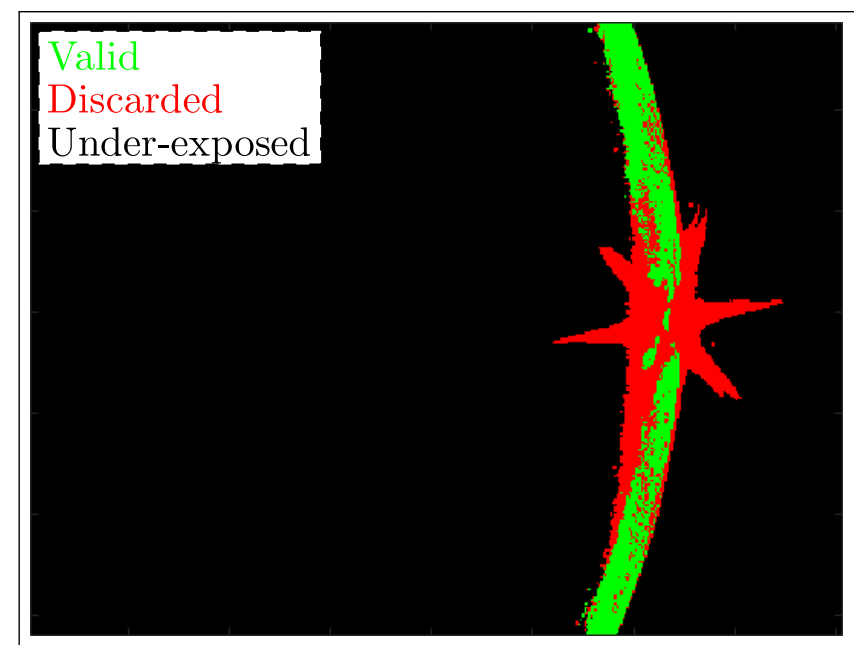

Figure 4: Output mask of the diffraction removal algorithm. Pixels affected by the diffraction are detected (in red) and therefore, can be discarded from the measurement.

cording to the authors, $\mathscr{K}$ is defined as the remaining diffraction blur that the algorithm is not able to separate. In particular, for a given image $I$ without diffraction, if the acquired picture with diffraction is $I \otimes P S F$, then the algorithm outputs $I \otimes \mathscr{K}$. The main interest is that $\mathscr{K}$ is a lot smaller than the PSF kernel. Therefore, the range of the blur is significantly reduced.

However, this residual kernel is defined for a worst-case scenario picture. This means that in the general case the $\mathscr{K}$ kernel is over-estimated and, consequently, the meaningful effective kernel is exaggerated for a given pixel. For instance, if the image is locally flat, the blurring from diffraction has no effect. Therefore, even if this remaining diffraction effect in an image seems to prevent an accurate measurement, we can argue that considering that diffraction ranges as far as is the $\mathscr{K}$ kernel is only valid in extreme conditions.

\section{Material Non-Uniformity}

To process the acquired data, the setup model needs the assumption that the sample sphere is covered with a uniform material, in other words, the BRDF function is not varying across the sample surface. There are two different effects that can slightly violate this assumption: the white light speckle and the manufacturing process of the sample.

The white light speckle (cf. Fig. 3) induces small colored sparkles, due to the spatial coherence of the light (counterpart of a good collimation) that makes light diffract on the small local random roughnesses of the sample. In order to correct for this effect, one can notice that averaging different realizations of the measurement converges to the same result as if we had an incoherent light source. Therefore, we choose to repeat each BRDF measurement several times (typically three to five times) while rotating the sample by a small angle between two measurements. With this technique, a given pixel of the camera corresponds, on the sample's surface, to a different realization of the random roughness that disappears when averaging.

The material non-uniformity emerges from the manufacturing process limitations. Even if the covering material of the sphere is chemically uniform, the surface quality is not always ideal (mostly scratches). For very diffuse materials, these scratches are not visible and are irrelevant. However, for more specular material, scratches have the tendency to offset the main specular direction and to add secondary specular directions. To address this issue, the same averaging method is applied as for eliminating the speckle effect. Indeed, by averaging through various surface parts seen from the same viewpoint, the scratches get blurred away. The more specular a material is, the more it will be necessary to repeat the measurement to blur the effects of scratches.

Regarding the configurations removed by the diffraction detection algorithm, in order to remain conservative, if any repeated BRDF configuration is considered polluted by diffraction, therefore the average also.

\section{Uncertainty Model}

Uncertainty values are included to guarantee the reliability of our measurement. The BRDF values and configurations are given within boundaries, tracked all along the calibration and measurement process from various original sources of error.

\section{Propagation of Errors}

As shown in Figure 2, uncertainties can be backtracked to the different manufacturer uncertainties (such as the radius of the sphere, the repeatability of the rotation arm,...), to the setup construction limitations (such as the collimation quality, numerical aperture of the camera lens,...) and to the various errors inherent in the calibration process. The uncertainties, chosen symmetrical around the measured values, are computed using the classical error propagation method. Considering a set of $n$ parameters $\mathbf{x}=\left\{x_{i}\right\}_{i \in[1, n]}$ with its corresponding set of uncertainty values $\left\{\Delta x_{i}\right\}_{i \in[1, n]}$, computing a new parameter $y=F(\mathbf{x})$ gives a corresponding error

$$
\Delta y=\sqrt{\sum_{i=1}^{n}\left(\frac{\partial F(\mathbf{x})}{\partial x_{i}} \Delta x_{i}\right)^{2}} .
$$

In our particular setup, correlations between the uncertainties are neglected and not added into equation (3). This is due to the fact that determining a particular parameter involves a lot of predetermined parameters and a calibration method. Therefore, the particular correlation between two parameters will be blurred within the noise brought by every other source of error [12].

\section{Diffraction Convolution Kernel}

Despite the track on uncertainties, imaging techniques come with convolutional operators (blur from out-of-focus, diffraction,...). These convolutions do not perturb directly the pixel values but spread them. Consequently, we cannot be sure that, in reality, the value of a pixel is not given by one or several of its neighbors. Therefore, the size of the convolution kernel gives a supplementary uncertainty on the actual pixel position, that has to be included into our analysis. One major concern is how should we get the circle of confusion (COC) given the kernel.

In our setup, the only important convolution is caused by diffraction, since a HDR measurement can lead to a very wide convolution kernel. Even though the diffraction detection algorithm is applied, the residual kernel can be still wide (40 pixels wide in our setup). To put things into context, a $6 \mathrm{~cm}$ diameter spherical sample is seen as 800 pixels wide onto the sensor of our 


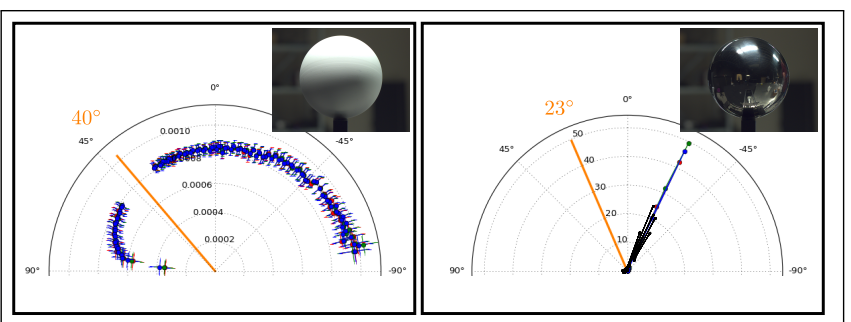

Figure 5: Incident plane BRDF measurements with uncertainty bars for the two extreme materials: diffuse (left) and specular (right). In the specular BRDF, the black curve represents the number of values detected as diffracted, they are clearly concentrated around the specular lobe.

camera, then a 40 pixels wide circle of confusion (COC) would lead to an extraordinary high uncertainty on the view direction. Indeed, it seems not realistic, and this corroborate the fact that the residual diffraction kernel is defined via a worst case scenario. More realistically, the size of the COC depends on the local image frequency content. For instance, if the image is locally very contrasted, such a high COC is justified since bright spots influence a lot their darkest neighbors. In contrary, if the image has a low frequency content, the $\mathrm{COC}$ can be almost reduced to zero. Knowing the realistic $\mathrm{COC}$ for each pixel would mean to be able to deconvolve diffraction from the signal, which is ill-posed and not desirable in a measurement context (cf. Lucat and al. [7]). As a consequence, for the sake of completeness, our measured BRDFs come with a file representing this diffraction kernel, letting future users the possibility to take it into consideration in the exploitation of the measurements.

\section{Results}

\section{Measurement Quality}

Due to the method, the measurement quality is very much dependent on the type of material. For this reason, we focus our conclusions on two extreme materials: a Spectralon $(\mathbb{R}$ material (very diffuse, white, cf. Fig. 5 left), and a mirror material (very specular, cf. Fig. 5 right).

Because our rotation stage can rotate almost continuously, it is possible to achieve very dense measurements. However, the quantity of interest is the uncertainty for each measured BRDF configuration. The angular uncertainties (cf. Fig 6) are very configuration-dependent, from confident angular configurations to unreliable values, mostly at grazing angles. Indeed, grazing angle configurations are close to the border of the sphere. In fact, because of the surface curvature, a small variation of the view or light directions leads to an important change of the local surface normal, and therefore an important change of the $\left(\theta_{i}, \theta_{o}, \Delta \phi\right)$ angles.

Given all the measured BRDF configurations for a given material, we choose to synthesize the achieved quality of our setup by referring to the median values. In fact, it appears that these particular values are representative of a large number of configurations, except from some extreme cases.

As shown in Table 3, the variations of the uncertainties across the different angular BRDF configurations generally lie around $1.2^{\circ}$. As expected, the $\theta_{i}$ and $\theta_{o}$ values become less reliable at grazing angles (cf. Fig. 6), and the $\Delta \phi$ angle is uncertain

\begin{tabular}{|c|c|c|}
\hline Quantity & Diffuse & Specular \\
\hline$\Delta \theta_{o}$ & $0.01^{\circ}(\mathrm{min}) / 1^{\circ}(\mathrm{med}) / 36^{\circ}(\max )$ \\
$\Delta \theta_{i}$ & \multicolumn{2}{|c|}{$0.5^{\circ} / 1.8^{\circ} / \infty^{\circ}$} \\
$\Delta \Delta \phi$ & $0.2^{\circ} / 1.7^{\circ} / \infty^{\circ}$ \\
\hline$\Delta\left(\rho \cos \left(\theta_{o}\right)\right)$ & $0.0001 \% / 0.1 \% / 0.6 \%$ \\
\hline $\begin{array}{c}\text { Repeatability } \\
\text { (out of 3 trials) }\end{array}$ & $0.04 \% / 1.6 \% / 83 \%$ & $0.01 \% / 5.5 \% / 140 \%$ \\
\hline $\begin{array}{c}\text { Amount of valid } \\
\text { measurements }\end{array}$ & $90 \%$ & $5.0 \%$ \\
\hline $\begin{array}{c}\text { Amount of } \\
\text { diffraction } \\
\text { configurations }\end{array}$ & $4.2 \%$ & $78 \%$ \\
\hline
\end{tabular}

Table 3: Statistical quantities to quantify the measurement quality and the impact of the diffraction removal algorithm. The percentage of diffraction configurations is only computed among the valid configurations. Non-valid measurements are mostly underexposed pixels that appear when the material dynamic range is higher than the camera one ( 7 decades in our setup).

around the north pole of the hemisphere. Regarding the BRDF values, we choose to quantify the quality of $\rho \cos \left(\theta_{o}\right)$ instead, since it is directly proportional to the measured pixel values. In this case, the uncertainties do not differ much between the two materials, falling below a maximum error of $1 \%$. This comes from the fact that the relative error on a pixel value is almost constant over the whole camera dynamic range. The error on $\rho$ then combines directly the error on $\rho \cos \left(\theta_{o}\right)$ with $\Delta \theta_{o}$, indeed leading to highly increasing uncertainties at grazing angles (cf. errors bars in Fig. 5).

The repeatability of our measurements depends on the type of material, it can reach $1.6 \%$ (median error) for diffuse materials to $5.5 \%$ for specular ones. In particular, as expected from our method that corrects the material non-uniformity, the more specular a material is, the more averaging of the signal is necessary. This number of needed repetitions goes unfeasibly high for the most specular materials. Thus, the repeatability of specular materials is considerably diminished.

As expected, the diffraction removal algorithm reduces drastically the number of measurement configurations with the material specularity. Depending on the type of material, this algorithm can remove from $4 \%$ up to $80 \%$ of all the valid (i.e., wellexposed) measured configurations, decreasing the interest for an image-based measurement. Of course, these diffraction configurations are situated beside the brightest BRDF configurations, in particular next to the specular lobe (cf. Fig. 5, right plot - black curve). Therefore, when measuring very specular materials, we would rather advise to use more specialized setups [13].

\section{Difficulties and Limitations}

Even if not within our focus, our method could be improved regarding the acquisition time. In particular, blurring the material non-uniformities require to repeat the measurement multiple times, and then multiplies the acquisition time. Furthermore, in its 

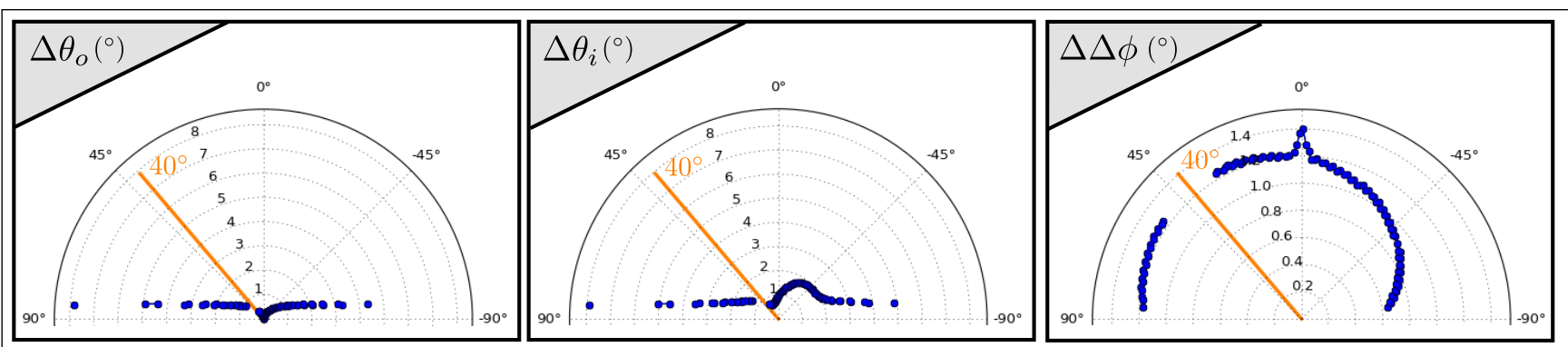

Figure 6: Incident plane uncertainties of the BRDF angular configurations (for a $40^{\circ}$ incident light direction). The uncertainties vary a lot depending on the angular configuration, highlighting the fact that the resolution is not absolute, but position-dependent.

current version, the diffraction removal algorithm is time consuming, taking about 60 s to process a single HDR image whereas the other post-processes only take a few seconds. However, the acquisition time remains in the order of magnitude of a few hours, as do the setups of which ours is inspired.

This type of acquisition method has also intrinsic limitations that constraint the usage of the BRDF measurements. As shown in Figure 5, a $14^{\circ}$ wide cone of data is missing in the retro-reflection direction. It arises from the design of our setup, when the camera passes in front of the light source. Moreover, the uncertainties on the BRDF values skyrocket at grazing angles, because of the $1 / \cos \left(\theta_{o}\right)$ multiplication needed to transform a pixel value into a BRDF value. Rather than discarding these configurations, we advise to use the $\rho \cos \left(\theta_{o}\right)$ values instead, which are much more reliable.

\section{Future Work}

Other BRDF setups focus on increasing the resolution or the acquisition time by changing the measurement technique or the hardware, we choose to focus on increasing the data reliability for a given hardware configuration. The first direction is to work on improving the diffraction treatment. One way is to take multiple images for different rotations of the camera around its optical axis (for instance, via a robot arm). Such a technique would virtually rotate the diffraction pattern, so that the discarded pixels change for each image. A final merge between all these images would lead to a result with less discarded pixels. In a future work, we may give a priori on the discarded pixels because of diffraction: we know for instance that diffraction mostly increases a given pixel value, so that its true value must be lower than the measured one. An other future work is to avoid repeating each measurement for blurring the material non-uniformity. For that purpose, it could be interesting to break the white speckle effect by erasing the light coherence with a spatial light modulator. If we want to decrease the measurement error, it is also possible to replace the camera CCD by one with smaller pixels. However, the intrinsic sphere curvature will always induce unreliable BRDF configurations at grazing angles. As a consequence, even if a spherical material is a good idea regarding the acquisition time compared to a planar sample, it will always be limiting regarding the BRDF measurement precision.

\section{Acknowledgments}

This project is supported by ANR Project Materials (ANR15-CE38-0005). R. Hegedus is grateful to the Alexander von Humboldt Foundation, and acknowledges their support for his fel- lowship for experienced researchers.

\section{References}

[1] Stephen R Marschner, Inverse Rendering for Computer Graphics, Computer, 1998, vol. 95, pg. 1295.

[2] Wojciech Matusik and al., Efficient Isotropic BRDF Measurement, Proc. 14th Eurographics workshop on Rendering, pg. 241. (2003).

[3] David Krik Mcallister, A Generalized Surface Appearance Representation for Computer Graphics, The University of North Carolina at Chapel Hill, 2002.

[4] Jason Lawrence and al., Inverse Shade Trees for Non-parametric Material Representation and Editing, ACM Papers on - SIGGRAPH '06, vol. 25, pg. 735. (2006).

[5] Peiran Ren and al., Pocket Reflectometry, ACM Transactions on Graphics, vol. 30, pg. 1. (2011).

[6] Addy Ngan and al., Experimental Analysis of BRDF Models, Proc. Eurographics Symposium on Rendering, vol. 2, pg. 117. (2005).

[7] Antoine Lucat and al., Diffraction Effects Detection for HDR Imagebased Measurements, Optics Express, 25, 27146 (2017).

[8] Fe Nicodemus and al., Geometrical Considerations and Nomenclature for Reflectance, Science And Technology, 60, 1 (1977).

[9] Szymon M. Rusinkiewicz, A New Change of Variables for Efficient BRDF Representation, Proc. Eurographics Symposium on Rendering, pg. 11. (1998).

[10] Jean-Yves Bouguet, Camera Calibration Toolbox, URL: http://www.vision.caltech.edu/bouguetj/calib_doc/

[11] T. Stewart McKechnie, Image-plane Speckle in Partially Coherent Illumination, Optical and Quantum Electronics, 8, 61 (1976).

[12] Ian Farrance and al., Uncertainty of Measurement: A Review of the Rules for Calculating Uncertainty Components through Functional Relationships, The Clinical biochemist, 33, 49 (2012).

[13] Gael Obein and al., Evaluation of the Shape of the Specular Peak for High Glossy Surfaces, International Society for Optics and Photonics, vol. 9018, pg. 901805. (2014).

\section{Author Biography}

Antoine Lucat received his engineering degree in Optics from the Institut d'Optique Graduate School (2015). During his two-years apprenticeship, he worked on the engineering of the petawatt laser PETAL (CEA, Bordeaux, France). Antoine is now a PhD student (2015-) in the MANAO team, which is born from the collaboration of the University of Bordeaux, the Institut d'Optique Graduate School and the INRIA. His work focuses on the study and acquisition of complex material appearances, based on imaging devices. 